\title{
A COUNTEREXAMPLE TO THE DEFORMATION CONJECTURE FOR UNIFORM TREE LATTICES
}

\author{
YING-SHENG LIU
}

(Communicated by Ron Solomon)

\begin{abstract}
Let $X$ be a universal cover of a finite connected graph. A uniform lattice on $X$ is a group acting discretely and cocompactly on $X$. We provide a counterexample to Bass and Kulkarni's Deformation Conjecture (1990) that a discrete subgroup $F \leq \operatorname{Aut}(X)$ could be deformed, outside some $F$-invariant subtree, into a uniform lattice.
\end{abstract}

A uniform tree $X$ is, by definition, the universal cover of a finite connected graph $Y$. A group $\Gamma<\operatorname{Aut}(X)$ is a uniform $X$-lattice if $\Gamma$ is discrete (i.e., every vertex stabilizer $\Gamma_{x}$ for $x \in V X$ is finite, where $V X$ is the set of all vertices of $X$ ) and the quotient graph $\Gamma \backslash X$ is finite. In this case $Y=\Pi \backslash X$, where $\Pi=\Pi_{1}(Y)$ is a free group acting freely on $X$. In particular $G \backslash X$ is finite, where $G=\operatorname{Aut}(X)$, and $\Pi$ is then a discrete cocompact subgroup (i.e., uniform lattice) in the locally compact group $G$.

The basic theory of such uniform three lattices was developed in BassKulkarni [BK]. In that paper they obtained many important results. It was shown there that if $X$ is a locally finite tree and $G=\operatorname{Aut}(X)$, then in order for $X$ to be uniform one needs not only the finiteness of $G \backslash X$ but also a 'unimodularity condition', that the locally compact group $G$ be unimodular; this condition also has a combinational interpretation. When $X$ is uniform they further showed that there is a uniform lattice $\Gamma \leq G$ such that $\Gamma \backslash X=G \backslash X$, that every free uniform lattice is conjugate to a subgroup of $\Gamma$, and that every uniform lattice $\Gamma^{\prime}$ is conjugate to one commensurable with $\Gamma$. Recall that $\Gamma_{0}$ and $\Gamma_{1}$ are said to be commensurable, denoted $\Gamma_{0} \sim \Gamma_{1}$, if the index $\left[\Gamma_{i}: \Gamma_{0} \cap \Gamma_{1}\right]$ is finite for $i=0,1$.

In [BK], one finds the Deformation Conjecture. This asserted, roughly, that a discrete subgroup $F \leq G$ could be 'deformed', outside some $F$-invariant subtree, into a uniform lattice. This conjecture was proved in [BK] whenever $G \backslash X$ is a tree or a loop. In this paper, we provide a counterexample to the Deformation Conjecture.

Let $X$ be a uniform tree, $G=\operatorname{Aut}(X)$.

Definition. Let $F \leq G$ and let $Y \subset X$ be an $F$-invariant subtree. By a deformation of $F$ outside of $Y$ we mean a monomorphism $h: F \rightarrow G$ such that

Received by the editors March 15, 1993 and, in revised form, April 21, 1993.

1991 Mathematics Subject Classification. Primary 05C25, 05 C05. 
$h(f)|Y=f| Y$ for each $f \in F$. We call $h$ a deformation of $F$ into $\Gamma \leq G$ if $h(F) \leq \Gamma$.

Deformation Conjecture ([BK, (4.20)]). Given a discrete group $F \leq G$ and an $F$-invariant subtree $Y$ such that $F \backslash Y$ is finite, we can deform $F$ outside $Y$ into a uniform $X$-lattice.

In [BK, (4.24)] it was verified whenever $X$ is homogeneous or when $G \backslash X$ is a tree. We show here, by an explicit example with $G \backslash X$ a triangle, that the Deformation Conjecture fails with $F$ a finite cyclic group.

Before giving the example, we shall recall briefly some concepts introduced in [B1] and [BK].

Let $X$ be a uniform tree, $\Gamma$ be a uniform $X$-lattice. Suppose that $A=\Gamma \backslash X$ is the quotient of $X$ modulo $\Gamma, p: X \rightarrow A$ is the canonical projection. Choose a subtree $S \subset X$ such that $p$ maps $S$ to $A$ bijectively on edges. Let $i: E A \rightarrow$ $\mathbf{Z}_{>0}$ be a function, such that $i(e)=\left[\Gamma_{\partial_{0} \tilde{e}}: \Gamma_{\tilde{e}}\right]$, where $\Gamma_{*}=\operatorname{Stab}_{\Gamma}(*), E A$ is the set of all edges of $A, e \in E A, \tilde{e} \in E S, p(\tilde{e})=e$, and $\partial_{0} \tilde{e}$ is the initial vertex of $\tilde{e}$. We call $(A, i)$ the edge-indexed graph of $X$ modulo $\Gamma$. Taking a base point $a \in A$, then, by [B], the universal cover of $(A, i)$ at a base point $a \in A$ is uniquely determined and is isomorphic to $X$. Moreover, $(A, i)$ is unimodular, i.e. (see [BK, (1.2), (1.3)]), for any closed edge path $\left(e_{1}, e_{2}, \ldots, e_{n}\right)$ in $A$,

$$
\prod_{j=1}^{n} i\left(e_{j}\right) / \prod_{j=1}^{n} i\left(\overline{e_{j}}\right)=1
$$

$\left(\partial_{0} \overline{e_{j}}=\partial_{1} e_{j}, \partial_{1} \overline{e_{j}}=\partial_{0} e_{j}\right.$, where $\partial_{1} e_{j}$ is the terminal vertex of $\left.e_{j}\right)$. Conversely, the universal cover of any unimodular edge-indexed finite graph $(A, i)$ at a fixed base point is a uniform tree. Now we can produce the counterexample.

Example. Consider the edge-indexed graph (cf. [B1, A]):

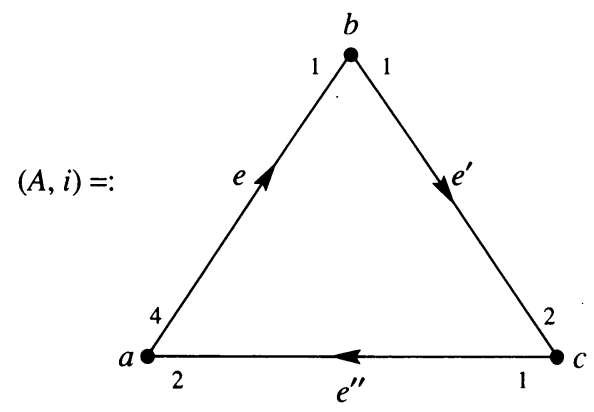

and its universal cover $X=(\widetilde{A, i}, a)$, with projection $p: X \rightarrow A$.

Since $\frac{4}{1} \cdot \frac{1}{2} \cdot \frac{1}{2}=1,(A, i)$ is unimodular and hence $X$ is a uniform tree. Put $G=\operatorname{Aut}(X)$.

Theorem. With $X$ as above, there exists a finite cyclic group $F \leq G$ which cannot be deformed outside a finite tree into a uniform $X$-lattice. 
Proof. Let $Y$ be the following finite subtree of $X$ :

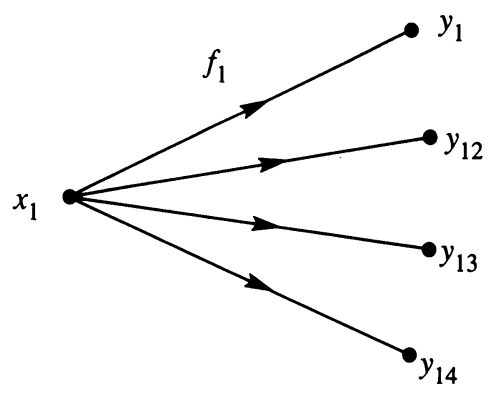

where $p\left(x_{1}\right)=a$ and all edges project to $e$. Choose $g \in G$ of order 3 with action on $Y$ given by: $g\left(x_{1}\right)=x_{1}, g\left(y_{1}\right)=y_{12}, g\left(y_{12}\right)=y_{13}, g\left(y_{13}\right)=y_{1}$, $g\left(y_{14}\right)=y_{14}$. This is possible since $p: X \rightarrow A$ maps the four edges of $Y$ to one edge $e$, i.e., the four edges are in the same $G$-orbit. Therefore, the four rooted subtrees which are components of $(X-Y)$ connecting to $y_{1}, y_{12}, y_{13}$, $y_{14}$ respectively are isomorphic to each other.

Suppose, on the contrary, that $F$ can be deformed outside $Y$ into a uniform $X$-lattice $\Gamma$. Hence, the exists an $\gamma \in \Gamma$ such that $g|Y=\gamma| Y$.

It is shown in [BK, (4.7)] that there is a uniform $X$-lattice $\Phi$ such that $\Phi \backslash X=G \backslash X$. And it is also shown in [BK, (4.15)] that any two uniform lattices are commensurable up to conjugacy. So there exists an $\alpha \in G$ such that $\Gamma$ and $\alpha \Phi \alpha^{-1}$ are commensurable. Put $\Phi^{\prime}=\alpha \Phi \alpha^{-1}$; then $\Gamma \sim \Phi^{\prime}$ and $\Phi^{\prime} \backslash X=G \backslash X$.

Choose a finite linear subtree $S \subset X$ starting with $f_{1}$ such that the canonical projection $p: X \rightarrow A=G \backslash X=\Phi^{\prime} \backslash X$ maps $S$ to $\Phi^{\prime} \backslash X$ bijectively on edges. Thus $S$ is a three-edge path, say $\left(f_{1}, f_{1}^{\prime}, f_{1}^{\prime \prime}\right)$, with vertex sequence $x_{1}, y_{1}$, $z_{1}, x_{2}$ as follows:

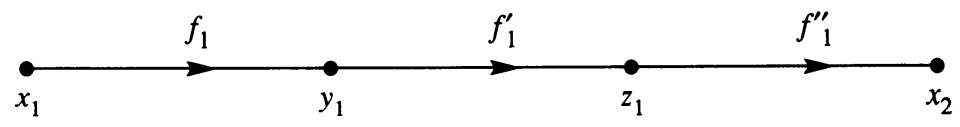

Thus $p\left(f_{1}\right)=e, p\left(f_{1}^{\prime}\right)=e^{\prime}, p\left(f_{1}^{\prime \prime}\right)=e^{\prime \prime}$, and $p\left(x_{2}\right)=a=p\left(x_{1}\right)$. Choose $\pi \in \Phi^{\prime}$, such that $x_{2}=\pi x_{1}$.

Since $\Gamma \sim \Phi^{\prime}$, i.e., $\left[\Phi^{\prime}: \Gamma \cap \Phi^{\prime}\right]<\infty$, there is an integer $k$, such that $\pi^{k} \in \Gamma$. Put $f_{2}=\pi f_{1}$. Then $\left(f_{1}, f_{1}^{\prime}, f_{1}^{\prime \prime}, f_{2}\right)$ is a reduced edge path. Indeed, $p\left(\overline{f_{1}^{\prime \prime}}\right)=\overline{e^{\prime \prime}} \neq e=p\left(f_{2}\right)$ implies $\overline{f_{1}^{\prime \prime}} \neq f_{2}$. Inductively, we can deduce that $Z=\bigcup_{i=0}^{k-1} \pi^{i} S$ is a reduced linear tree:

$Z$ :

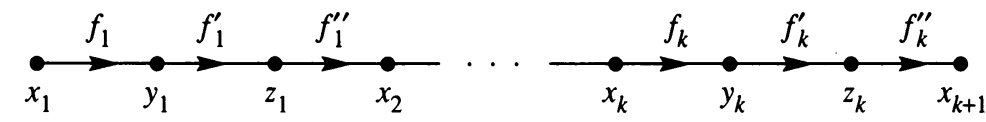

where $f_{i}=\pi^{i-1} f_{1}, f_{i}^{\prime}=\pi^{i-1} f_{1}^{\prime}, f_{i}^{\prime \prime}=\pi^{i-1} f_{1}^{\prime \prime}, i=1,2, \ldots, k$. Thus, $x_{k+1}=\partial_{1} f_{k}^{\prime \prime}=\partial_{1} \pi^{k-1} f_{1}^{\prime \prime}=\pi^{k-1} \partial_{1} f_{1}^{\prime \prime}=\pi^{k-1} x_{2}=\pi^{k} x_{1}$. As $\pi^{k} \in \Gamma$,

$$
\Gamma_{x_{k+1}}=\Gamma_{\pi^{k} x_{1}}=\pi^{k} \Gamma_{x_{1}} \pi^{-k} \text {. }
$$


Since $p\left(f_{i}\right)=p\left(\pi^{i-1} f_{1}\right)=e, p\left(f_{i}^{\prime}\right)=p\left(\pi^{i-1} f_{1}^{\prime}\right)=e^{\prime}, p\left(f_{i}^{\prime \prime}\right)=p\left(\pi^{i-1} f_{1}^{\prime \prime}\right)$ $=e^{\prime \prime}$, by the indices of $(A, i)$, we have $\left[G_{x_{i+1}}: G_{f_{i}^{\prime \prime}}\right]=2,\left[G_{x_{i}}: G_{f_{i}^{\prime}}\right]=2$, $\left[G_{y_{i}}: G_{f_{i}}\right]=1, i=1,2, \ldots, k$.

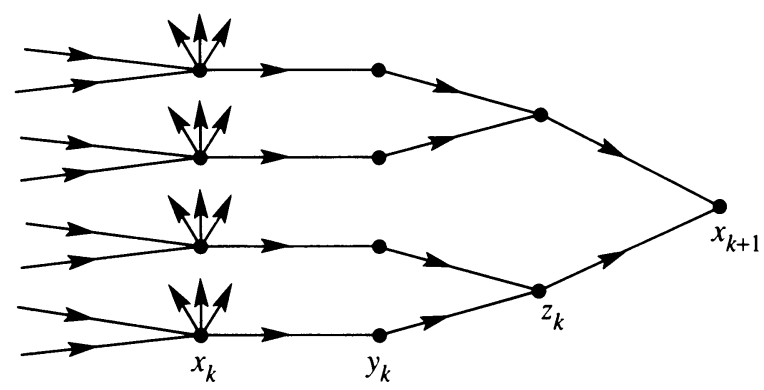

This implies that for each $\alpha \in G_{x_{k+1}}, \alpha^{2}$ fixes $z_{k}, \alpha^{4}$ fixes $y_{k}$ and $x_{k}$, and, inductively, $\alpha^{4^{k}}$ fixes $x_{1}$. Then for each $\beta \in \Gamma_{x_{k+1}}$,

$$
\beta^{4^{k}} \in \Gamma_{x_{1}}
$$

Recall that $\gamma \in \Gamma_{x_{1}}$ has the same action on subtree $Y$ as $g$ does; it follows that

(3) $\quad \gamma^{2^{l}}$ does not fix $f_{1}$ for any $l \in \mathbf{Z}$ and, hence, does not fix $x_{k+1}$.

Putting $\gamma_{0}=\gamma$, we may inductively define

$$
\gamma_{i}=\left(\pi^{k} \gamma_{i-1} \pi^{-k}\right)^{4^{k}}, \quad i=1,2,3, \ldots
$$

We claim that for each $j=1,2,3, \ldots$

(a) $\gamma_{j}=\pi^{j k} \gamma_{0}^{4^{j k}} \pi^{-j k}$,

(b) $\gamma_{j} \in \Gamma_{x_{1}} \cap \Gamma_{x_{k+1}}$ for $j>0$,

(c) $\gamma_{j} \neq \gamma_{i}$, if $j \neq i$.

We prove the claim by induction.

For $j=1$, by the definition of $\gamma_{j}, \gamma_{1}=\left(\pi^{k} \gamma_{0} \pi^{-k}\right)^{4^{k}}=\pi^{k} \gamma_{0}^{4^{k}} \pi^{-k}$, whence (a). Since $\gamma_{0}=\gamma \in \Gamma_{x_{1}}$, it follows from (1) that $\pi^{k} \gamma_{0} \pi^{-k} \in \Gamma_{x_{k+1}}$. By (2), $\left(\pi^{k} \gamma_{0} \pi^{-k}\right)^{4^{k}} \in \Gamma_{x_{1}}$, whence (b). By (3), $\gamma_{0} \notin \Gamma_{x_{k+1}}$, but $\gamma_{1}=\left(\pi^{k} \gamma_{0} \pi^{-k}\right)^{4^{k}} \in$ $\Gamma_{x_{k+1}}, \gamma_{0} \neq \gamma_{1}$, whence (c).

Assume that the claim is true for some $j>0$. Then

(a) $\gamma_{j+1}=\left(\pi^{k} \gamma_{j} \pi^{-k}\right)^{4^{k}}=\pi^{k} \gamma_{j}^{4^{k}} \pi^{-k}=\pi^{k}\left(\pi^{j k} \gamma_{0}^{4^{j k}} \pi^{-j k}\right)^{4^{k}} \pi^{-k}=$ $\pi^{k} \pi^{j k} \gamma_{0}^{4^{j k} 4^{k}} \pi^{-j k} \pi^{-k}=\pi^{(j+1) k} \gamma_{0}^{4(j+1) k} \pi^{-(j+1) k}$.

(b) Since $\gamma_{j+1}=\left(\pi^{k} \gamma_{j} \pi^{-k}\right)^{4^{k}}$ and, by the induction assumption, $\gamma_{j} \in \Gamma_{x_{1}} \cap$ $\Gamma_{x_{k+1}}$, it follows, by (1), that $\pi^{k} \gamma_{j} \pi^{-k} \in \Gamma_{x_{k+1}}$. Therefore, by (2), $\left(\pi^{k} \gamma_{j} \pi^{-k}\right)^{4^{k}} \in$ $\Gamma_{x_{1}} \cap \Gamma_{x_{k+1}}$.

(c) Suppose that $\gamma_{m}=\gamma_{n}$ for some $m>n$. Then, by (a), we have

$$
\pi^{m k} \gamma_{0}^{4^{m k}}=\pi^{-m k}=\pi^{n k}{\gamma_{0}^{4 k}}^{-n k}
$$

then

$$
\pi^{(m-n) k} \gamma_{0}^{4^{m k}} \pi^{-(m-n) k}=\gamma_{0}^{4^{n k}}
$$


i.e., $\left(\pi^{(m-n) k} \gamma_{0}^{4(m-n) k} \pi^{-(m-n) k}\right)^{4^{n k}}=\gamma_{0}^{4^{n k}}$. Then the left side is $\gamma_{m-n}^{4^{n k}}, m-n>0$. By (b), $\gamma_{m-n} \in \Gamma_{x_{k+1}}$, so does $\gamma_{m-n}^{4^{n k}}$. But, by (3), the right side $\gamma_{0}^{4^{n k}} \neq \Gamma_{x_{k+1}}$. Thus we proved the claim.

Therefore, we have infinitely many $\gamma_{i} \in \Gamma_{x_{1}}, i=1,2, \ldots ;$ but $\Gamma_{x_{1}}$ is a finite subgroup. This contradiction tells us that $f=\langle g\rangle$ cannot be deformed outside $Y$ into a uniform $X$-lattice.

We have also prove the following Corollary.

Corollary. The union of all uniform $X$-lattices is not dense in $G$.

Indeed, in the example above, for $g \in G$ and $Y \subset X$, there is no uniform $X$-lattice $\Gamma$ and $\gamma \in \Gamma$, such that $\gamma|Y=g| Y$. This means that $g$ is isolated from the union of all uniform $X$-lattices.

However, it is proved in [L1] that the commensurability group $C_{G}(\Gamma)=$ $\left\{g \in G \mid g \Gamma g^{-1} \sim \Gamma\right\}$ is dense in $G$. More precisely, the group generated by all uniform $X$-lattices commensurable with $\Gamma$ is dense in $G$. (See [L2].)

\section{ACKNOWLEDGMENT}

The above results formed a portion of my doctoral dissertation [L3]. I am very grateful to my advisor Hyman Bass for all of his help and encouragement. He has always been concerned about my work and generous with his time and ideas.

I also wish to thank Ravi Kulkarni, John Morgan, and Gouliang Yu for their interest and helpful comments. Finally, I would like to thank the referee for his careful reading and good suggestions.

\section{REFERENCES}

[AB] R. Alperin and H. Bass, Length functions of group actions on $\Lambda$-trees, Combinatorial Group Theory and Topology, Ann. of Math. Stud., no. 111, Princeton Univ. Press, Princeton, NJ, 1987, pp. 265-378.

[B1] H. Bass, Covering theory for graphs of groups, J. Pure Appl. Algebra (to appear).

[B2] _ Group actions on non-archimedean trees, Arboreal Group Theory (Roger C. Alperin, ed.), Math. Sci. Res. Inst. Publ., vol. 19, Springer-Verlag, Berlin and New York, 1988, pp. 69-131.

[BK] H. Bass and R. Kulkarni, Uniform tree lattices, J. Amer. Math. Soc. 4 (1990), 843-902.

[K] R. Kulkarni, Lattices on trees, automorphism of graphs, free groups, surfaces, preprint, CUNY, September 1988.

[KPS] A. Karass, A. Pietrowski, and D. Solitar, Finite and infinite cyclic extension of free groups, Austral. Math. Soc. 16 (1973), 458-466.

[L1] Y. Liu, Density of the commensurability groups of uniform tree lattices, J. Algebra (to appear).

[L2] _ A necessary condition for an elliptic element to belong to a uniform tree lattice, Proc. Amer. Math. Soc. (to appear).

[L3] _ Commensurability groups of uniform tree lattices, Columbia Univ. Dissertation, 1991.

[Lub] A Lubotzky, Trees and discrete subgroups of Lie groups over local fields, Bull. Amer. Math. Soc. (N.S.) 20 (1988), 27-31.

[S] J.-P. Serre, Trees, Springer-Verlag, New York, 1980.

Department of Mathematics, State University of New York, College at PlattsBURgh, Plattsburgh, New York 12901

E-mail address: liuysesplava.cc.plattsburgh.edu 